\title{
Habilidades sociais, estratégias de aprendizagem e desempenho acadêmico de alunos participantes e não participante do PIBID
}

Social skills, learning strategies and academic performance of participating students and nonparticipant PIBID

\author{
R. S. Lopes ${ }^{1}$; F.M. Versuti ${ }^{2 *}$ L. C. S. Elias ${ }^{3}$; M. R. G. Zanini-Correia ${ }^{4}$ \\ 1,2,3 Departamento de Psicologia//Laboratório de Psicologia da Educação e Escolar-Lapees, FFCLRP/USP, 14040- \\ 901, Ribeirão Preto-SP, Brasil
}

${ }^{4}$ Departamento de Psicologia/Centro Universitário das Faculdades Associadas de Ensino- UNIFAE, 13870-377 São João da Boa Vista- SP, Brasil

*versuti_stoque@ffclrp.usp.br

(Recebido em 21 de setembro de 2016; aceito em 19 de abril de 2017)

\begin{abstract}
O presente trabalho objetivou investigar diferenças e semelhanças no repertório social, nas estratégias de aprendizagem e no desempenho acadêmico de alunos que participaram e que não participaram do Programa Institucional de Bolsas de Iniciação à Docência (PIBID), subprojeto interdisciplinar das áreas de Ciências e Biologia. O PIBID tem seu desenvolvimento delineado por meio das Sequências de Ensino Investigativo (SEI), as quais podem ser vistas como um contribuinte para o processo de alfabetizar cientificamente. Para o desenvolvimento do presente estudo foram utilizados e aplicados dois instrumentos para a coleta de dados, o Inventário de Habilidade Social para Adolescentes (IHSA) [7], a Escala de Avaliação das Estratégias de Aprendizagem para o Ensino Fundamental (EAVAP-EF) [2] e para avaliação do desempenho acadêmico foram analisadas notas de uma avaliação em processo (AAP). A amostra foi composta por: estudantes do $6^{\circ}$ e $7^{\circ}$ anos não participantes e participantes do PIBID, respectivamente. Os resultados apontaram relações existentes entre variáveis mapeadas relativas a habilidades sociais, estratégias de aprendizagem e desempenho acadêmico. Observou-se assim a importância da estruturação de programas de intervenção que articulem as Habilidades Sociais, as Estratégias de Aprendizagem e propostas inovadoras de ensino, tais como o PIBID, de modo que possam atuar como fatores de proteção ao desenvolvimento dos alunos.
\end{abstract}

Palavras-chave: desempenho acadêmico, estratégias de aprendizagem, habilidades sociais.

This work it is a study that craved compare students participating and not participating in the Institutional Scholarship Program Introduction to Teaching (PIBID) in relation to variables: social skills, learning strategies and academic achievement in elementary school students, who attended the 6th or 7th year. The PIBID has outlined development through sequences of Investigative Education (SEI), which may be regarded as a contributor to the process of scientifically literate. For the development of this study were used and applied two instruments for data collection, the Inventory of Social Skills for Teens (IHSA) [7], the Evaluation Scale of Learning Strategies for Teaching fundamental (EAVAP-EF) [2] and evaluation of academic performance were analyzed notes of a process evaluation (AAP). Students who participated in the study were students of the 6th year non-participants and students from 7 participants Pibid. The results were placed in a spreadsheet and subjected to statistical analysis, which identified links between mapped variables related to social skills, learning strategies and academic performance. The mapping of variables performed indicated the importance of structuring intervention programs that link the Social Skills, the Learning Strategies and innovative teaching proposals, such as PIBID, so that they can act as protective factors to the development of students. Insert the abstract here.

Keywords: academic achievement; learning strategies; social skills.

\section{INTRODUÇÃO}

Devida à importância mundial do processo educativo para o desenvolvimento da sociedade, deparamos constantemente com ações e informações referentes ao desempenho escolar, com o 
debate sobre o ensino tradicional e novas formas de aprendizagem com uso de estratégias diferenciadas.

Vivenciamos tempos de globalização, avanço tecnológico e científico que dão acesso a uma grande quantidade de informação, destacando no cenário educacional a importância do ensino de ciências. Diretrizes nacionais e internacionais apontam, a necessidade de que o ensino de Ciências considere o crescente impacto das evoluções científicas e tecnológicas e abordem em sala de aula temas mais próximos à realidade dos estudantes [1].

Nas últimas décadas, foram criadas e implantadas uma série de propostas visando a transformação do ensino, e, o Programa Institucional de Bolsa de Iniciação à Docência (PIBID) faz parte dessas ações do governo federal para a realização do Programa de Desenvolvimento da Educação, "integrando-se ao conjunto de reformas iniciadas em 2001, com a promulgação das Diretrizes Nacionais para a Formação, em nível superior, de Professores para a Educação Básica (Parecer CNE/CPn ${ }^{\circ} 009 / 2001$ e Resolução CNE/CP n¹/2002)", apresentando, assim, um conjunto de objetivos robustos e que visa combater problemas centrais relacionados à formação e à valorização do docente para a escola básica [4].

O subprojeto do PIBID das áreas de Biologia e Ciências contemplam como um dos seus objetivos principais, as discussões sobre as práticas escolares presentes nos currículos de Ciências e Biologia numa perspectiva da Alfabetização Científica, bem como a reformulação dessas práticas sob uma perspectiva criativa, investigativa e interdisciplinar. Tendo como meta, o desenvolvimento da linguagem e da argumentação científica, entendendo-as como centrais para a promoção da alfabetização científica, por meio de atividades de caráter investigativo, chamadas de Sequências de Ensino Investigativo, as SEI's, de maneira que traduza os procedimentos e características da prática científica.

O ensino por investigação fomenta o questionamento, o planejamento, a recolha de evidências, as explicações com bases nas evidências e a comunicação. Usa processos da investigação científica e conhecimentos científicos, podendo ajudar os alunos a aprender a fazer ciência e sobre ciência.

Existe uma preocupação crescente, em colocar a Alfabetização Científica com objetivo central do ensino de Ciências em toda a formação básica, o que encontra respaldo e consistência na percepção da necessidade emergente de formar alunos para atuação na sociedade atual, largamente cercada por artefatos da sociedade científica e tecnológica [15].

Pensando em fatores que influenciam o desempenho escolar, são relevantes as interações sociais no contexto educacional. A literatura tem apontado a existência de um amplificado reconhecimento da dimensão social dos processos de desenvolvimento e de aprendizagem colocando em evidência o papel das interações sociais e da comunicação na construção do conhecimento e da subjetividade E neste sentido, pesquisas [5] tem evidenciado que crianças mais habilidosas socialmente tendem a apresentar melhor desempenho acadêmico. Esta premissa justifica o planejamento de programas dedicados à promoção de habilidades sociais no contexto escolar [14].

O amplificado reconhecimento dos processos de desenvolvimento e de aprendizagem tem colocado em evidência o papel das interações sociais e da comunicação na construção do conhecimento e da subjetividade, podendo neste contexto situar o desenvolvimento interpessoal, observando-se atualmente um crescente estudo na interface entre Habilidades sociais e Educação[6].

Muitos trabalhos têm contribuído por ter documentado na literatura uma correlação significativa entre dificuldade de aprendizagem e déficits em habilidades sociais [8], evidenciando-se, assim, a presença de questões comportamentais ao ajustamento escolar do aluno em um sentido amplo, envolvendo tanto aspectos acadêmicos como interpessoais.

Outro aspecto relevante para o desempenho acadêmico são as estratégias de aprendizagem utilizadas pelos alunos, ou seja, sequências de procedimentos ou atividades que se escolhem como propósito de facilitar a aquisição, o armazenamento e a utilização da informação [12]. Resultados de pesquisas revelam que a intervenção para o desenvolvimento de estratégias de aprendizagem tem sido bem sucedida, de modo geral, pois é capaz de produzir, tanto uma melhora imediata em seu uso, quanto no rendimento escolar geral dos alunos [10]. 
Dada à relevância desses aspectos, o presente estudo buscou investigar diferenças e semelhanças no repertório social, nas estratégias de aprendizagem e no desempenho acadêmico de alunos que participaram e que não participaram do PIBID subprojeto interdisciplinar das áreas de Ciências e Biologia. Também pretendeu descrever a classificação obtida por cada grupo, de tais repertórios, considerando parâmetros inferior, médio e superior, estabelecidos pelos autores dos instrumentos.

\section{MATERIAL E MÉTODOS}

Trata-se de um estudo quantitativo, com delineamento transversal e comparativo. No que tange aspectos éticos, a participação dos alunos foi devidamente regulamentada pela assinatura do responsável no Termo de Consentimento Livre e Esclarecido e Termo de assentimento aos participantes, conforme prevê a resolução 466/12 do Conselho Nacional de Pesquisa.

\section{Participantes}

Trata-se de uma amostra de conveniência composta por 30 alunos, com idade média de 13 anos, sendo 21 meninas e 9 meninos, que cursavam o $6^{\circ}(n=15)$ ou $7^{\circ}$ ano $(n=15)$ do Ciclo II do Ensino Fundamental de uma escola estadual localizada em município de médio porte no interior do estado de São Paulo. Destaca-se que a escola participa do PIBID desde março de 2014, e sua escolha se deu através de sorteio, dentro de um conjunto de unidades participantes do programa.

Os alunos do $6^{\circ}$ ano compuseram o G1 - alunos não participantes do PIBID, subprojeto interdisciplinar das áreas de Ciências e Biologia, e os alunos do $7^{\circ}$ ano, G2 - alunos participantes do PIBID, subprojeto interdisciplinar das áreas de Ciências e Biologia.

\section{Materiais}

- Inventário de Habilidades Sociais para Adolescentes - IHSA [7]. Instrumento, composto por 38 itens, trata-se de um instrumento de auto-relato, referenciado à norma, que permite avaliar o repertório de habilidades sociais de adolescentes em um conjunto de situações interpessoais cotidianas, permitindo a identificação de déficits e recursos em classes e subclasses de habilidades sociais de adolescentes, que são: Empatia, Autocontrole, Civilidade, Assertividade, Afetividade e Desenvoltura Social.

- Escala de Avaliação de Estratégias de Aprendizagem para o Ensino Fundamental -EAVAPEF [10], composta por 31 questões que abordam estratégias utilizadas em situações de estudo e aprendizagem. São consideradas Estratégias de Aprendizagem, Estratégias Cognitivas, Estratégias metacognitivas e Ausência de estratégias metacognitivas disfuncionais.

- Desempenho acadêmico: foram analisadas as notas referentes a uma Avaliação em Processo, realizada pelo professor, seguindo um protocolo proposto pela escola.

\section{Procedimento}

Inicialmente foi feito contato com a escola e com os responsáveis pelos alunos para obtenção das autorizações e recolha de assinatura dos Termos de Consentimento Livre e Esclarecido. Em seguida, foram agendados com a coordenação e professores os dias e horários das aplicações dos instrumentos, que para ambos os grupos foi coletiva, embora cada aluno preencheu os instrumentos individualmente. Alunos do G1 ( $7^{\circ}$ ano) foram avaliados separadamente de G2 $\left(6^{\circ}\right.$ ano). Cada aplicação durou em torno de XX minutos. As avaliações de desempenho foram obtidas através de consulta a documentos cedidos pela escola.

\section{Análise de dados}

Para a análise de dados os instrumentos foram cotados conforme a orientação de seus autores o e foi utilizado o programa SPSS - IBM Statistics - versão 19. Foram realizadas estatísticas descritivas, de comparação entre grupos, se utilizando do teste $t$ de Student, quando as variáveis apresentaram distribuição normal, e o alternativo não paramétrico (Man-Whitney) quando este pressuposto não foi cumprido. O teste do Qui-quadrado foi aplicado para comparar a distribuição entre os dois grupos no que se refere a classificação: inferior, médio inferior, médio, médio 
satisfatório e superior, obtidas paras as variáveis. O valor de significância estatística considerado foi de $\mathrm{p} \leq 0,05$.

\section{RESULTADOS E DISCUSSÃO}

Os resultados das comparações entre os dois grupos estão dispostos na Tabela 1 e de acordo com as análises estatísticas foram significativas as diferenças observadas em: Autocontrole $(U=$ 44.00; $\mathrm{p}=0.004)$, Civilidade $(t=3.13 ; \mathrm{p}=0.005)$, Desenvoltura Social $(t=2.56 ; \mathrm{p}=0.016)$, EAVAP $(t=2.61 ; \mathrm{p}=0.014)$ e Ausência de estratégias $(\mathrm{t}=2.98 ; \mathrm{p}=0.008)$, sendo que G1 apresentou maiores médias nestas variáveis.

Tabela 1: Média, desvio padrão, máximo e mínimo nas variáveis do estudo para alunos participantes e não participantes do PIBID

\begin{tabular}{lllll}
\hline & \multicolumn{3}{c}{ Grupo de alunos } \\
\cline { 2 - 5 } \multicolumn{1}{c}{ Variáveis } & \multicolumn{2}{c}{$\begin{array}{c}\text { G1 } \\
\text { (n=15) }\end{array}$} & \multicolumn{2}{c}{$\begin{array}{c}\text { G2 } \\
(\mathbf{n = 1 5})\end{array}$} \\
\cline { 2 - 5 } & \multicolumn{1}{c}{ Média } & DP & Média & DP \\
\hline Habilidades Sociais & 98.47 & 15.34 & 89.00 & 20.74 \\
Empatia & 28.27 & 3.67 & 25.60 & 8.01 \\
Autocontrole & $20.33^{\mathrm{b}}$ & 5.51 & $13.80^{\mathrm{a}}$ & 6.62 \\
Civilidade & $20.33^{\mathrm{b}}$ & 2.22 & $16.27^{\mathrm{a}}$ & 4.51 \\
Assertividade & 17.40 & 5.50 & 14.73 & 4.59 \\
Afetividade & 11.93 & 3.71 & 11.47 & 5.12 \\
Desenvoltura Social & 11.47 & 3.72 & 7.93 & 3.82 \\
EAVAP & $32.80^{\mathrm{b}}$ & 9.94 & $25.40^{\mathrm{a}}$ & 6.37 \\
Ausência.de Estratégias & $13.93^{\mathrm{b}}$ & 6.80 & $8.33^{\mathrm{b}}$ & 2.61 \\
Metacognitivas.Disfuncionais & 8.33 & 2.61 & 9.40 & 3.96 \\
Estratégias. Cognitivas & 9.46 & 2.19 & 8.66 & 2.74 \\
Estratégias Metacognitivas & 55.76 & & 50.37 & \\
Desempenho Acadêmico & & & & \\
\hline
\end{tabular}

Nota: G1 - Alunos do $7^{\circ}$ ano, participantes do PIBID, G2 - Alunos do $6^{\circ}$ ano, não participantes do PIBID; DP - Desvio padrão; EAVAP - Escala de Avaliação de Estratégias de Aprendizagem para o Ensino Fundamental. Letras diferentes indicam diferenças significativas entre as médias nos testes estatísticos $\operatorname{com} p \leq 0,05$.

Na Tabela 2 se observa a distribuição da classificação dos percentis obtidos pelos grupos nos totais das Habilidades Sociais e EAVAP. O teste do Qui-quadrado indicou semelhanças na distribuição entre os grupos no que se refere às Habilidades Sociais $\left(\chi^{2}(4)=3,200, p=0,525\right)$ e a $\operatorname{EAVAP}\left(\chi^{2}(4)=3,200, p=0,063\right)$.

Analisando os resultados do grupo 1, percebeu-se que os participantes apresentaram um bom repertório médio de habilidades segundo o sistema de categorias de classificação de percentis. As subescalas dos grupos apresentaram resultados que representa um bom repertório com resultados dentro da média, com exceção da subescala Desenvoltura Social, que apresentou média inferior as demais, o que pode sugerir um acompanhamento ou um programa de intervenção já que a literatura esclarece que elas são comportamentos passíveis de serem aprendidos, sendo essas habilidades importantes em situação de exposição social e conversação, como apresentação de trabalhos em grupo, como pedir informações, explicar tarefas a colegas, conversar com pessoas de autoridades, habilidades importantes para a tendência atual do processo educativo, em formar um aluno ativo no seu processo de aprendizagem e para a construção de conhecimento significativo.

Já no grupo 2, a classificação alcançada, referente ao total geral do repertório de Habilidades Sociais, representam habilidades sociais médio inferior, já em relação as subescalas Assertividade, a classificação 1, médio inferior, demonstra um déficit na capacidade de lidar com situações interpessoais que demandam a afirmação e defesa de direitos e autoestima, com risco 
potencial de reação indesejável por parte do interlocutor (possibilidade de rejeição, de réplica ou de oposição), com a mesma classificação 1, a subescala Desenvoltura Social apresentaram resultado médio inferior o que mostra um déficit e problemas como os já relatados para o grupo 1 .

Tabela 2: Tabela cruzada entre grupos e classificação do percentil das Habilidades Sociais Média

\begin{tabular}{|c|c|c|c|c|}
\hline Variáveis & Percentil & $\begin{array}{c}\text { G1 } \\
(n=15)\end{array}$ & $\begin{array}{c}\text { G2 } \\
(n=15)\end{array}$ & Total \\
\hline Habilidades & Inferior & 3 & 6 & 9 \\
\hline \multirow[t]{5}{*}{ Sociais } & Médio inferior & 3 & 3 & 6 \\
\hline & Médio & 6 & 3 & 9 \\
\hline & $\begin{array}{l}\text { Médio } \\
\text { Satisfatório }\end{array}$ & 0 & 1 & 1 \\
\hline & Superior & 3 & 2 & 5 \\
\hline & Total & 15 & 15 & 30 \\
\hline \multirow[t]{6}{*}{ EAVAP } & Inferior & 6 & 13 & 19 \\
\hline & Médio inferior & 1 & 1 & 2 \\
\hline & Médio & 5 & 0 & 5 \\
\hline & $\begin{array}{l}\text { Médio } \\
\text { Satisfatório }\end{array}$ & 1 & 0 & 1 \\
\hline & Superior & 2 & 1 & 3 \\
\hline & Total & 15 & 15 & 30 \\
\hline
\end{tabular}

Nota: G1 - Alunos do $7^{\circ}$ ano, participantes do PIBID, G2 - Alunos do $6^{\circ}$ ano, não participantes do PIBID; EAVAP - Escala de Avaliação de Estratégias de Aprendizagem para o Ensino Fundamental.

Salienta-se que o repertório de habilidades sociais, em sala de aula é importante, por fomentar a concorrência e manutenção de comportamentos adequados que possibilitam a interação dos alunos entre si e com a professora.

Em relação ao uso de Estratégias de Aprendizagem o resultado também foi médio inferior para todas subescalas, o que indica um baixo uso de estratégias de aprendizagem ou até mesmo que estejam sendo usadas de forma indevida.

Os resultados demonstraram tanto para o grupo 1 como para o grupo 2 que, de um modo geral, os estudantes relataram recorrer pouco a estratégias de aprendizagem no momento de estudo já que a média de pontos na escala foi de 29,50, classificação 1, médio inferior. Sobre esse aspecto, $[2,13]$ enfatizam que o uso das estratégias de aprendizagem facilita a aprendizagem, uma vez que viabiliza a aquisição e a posterior recuperação e uso da informação, bem como fomenta o bom desempenho acadêmico.

Utilizar as estratégias de aprendizagem de forma adequada, permite ao estudante adquirir, organizar e reter informações necessárias ao processo de aprendizagem e, desta forma, contribuir para o sucesso escolar, uma vez que possibilitam aos alunos o envolvimento em seu próprio processo de aprendizagem e consequente diversificação das formas de estudo [10].

As evidências obtidas na presente pesquisa corroboram os dados obtidos, uma vez que apontam que o bom desempenho acadêmico dos estudantes se relaciona com o uso de estratégias de aprendizagem no momento de estudo. Uma avaliação diagnóstica do uso de estratégias de aprendizagem poderia viabilizar programas interventivos que focariam o seu emprego e a sua utilização diversificada $[1,2,11]$.

O processo de construção do conhecimento se dá através de interações entre a criança e seu meio, e se a aprendizagem de conceitos e habilidades afeta o desenvolvimento social, parece razoável considerar a relação em Habilidades Sociais e Estratégias de Aprendizagem na formulação de propostas pedagógicas. 
Comparando-se o desempenho acadêmico dos grupos de alunos, os participantes do PIBID, sinalizaram índices inferiores ao esperado, confirmando dados da literatura e referendando a necessidade da intervenção.

Pesquisas acumuladas na área educacional revelam que a construção de conhecimento é um processo complexo que está associado a fatores psicológicos, sociais e afetivos os quais precisam ser trabalhados conjuntamente dentro do ambiente escolar, já que este é o ambiente ideal para se perceber e trabalhar essas variáveis.

O ensino de Ciências não deve se restringir à transmissão de conhecimentos, mas deve mostrar aos alunos a natureza e a prática científica, explorando, sempre que possível às relações existentes entre ciência, tecnologia, sociedade e meio ambiente, sob esta perspectiva a investigação pode ser um instrumento para se desenvolver habilidades e competências, promovendo a aprendizagem e construção de conhecimento, de forma a criar condições para que os alunos sejam inseridos na cultura científica. Diante da complexidade que envolve o processo de ensino e aprendizagem das Ciências, destaca-se a importância de relacionar variáveis que possam influenciar positivamente ou negativamente, impossibilitando que os alunos alcancem os objetivos almejados dentro da proposta do ensino de Ciências.

Por fim, observamos que a participação do grupo 2 no PIBID, não os relacionou positivamente com os resultados, mas há de se considerar que este estudo não fez um comparativo antes da intervenção do PIBID e após a intervenção, o que seria de suma importância para obtenção de resultados mais verídicos em relação a participação dos alunos em projetos de intervenção, mas acredita-se que programas como o PIBID fomentam o interesse dos alunos, promovendo aos alunos a oportunidade de construírem o conhecimento, entender melhor os problemas valorizando a autonomia do aluno.

\section{CONCLUSÃO}

O mapeamento das variáveis realizado neste estudo indicou a importância da estruturação de programas de intervenção que articulem as Habilidades Sociais, as Estratégias de Aprendizagem e propostas inovadoras de ensino, tais como o PIBID, de modo que possam atuar como fatores de proteção ao desenvolvimento dos alunos quando submetidos ao processo de construção de conhecimento em situações tão complexas como as apresentadas em sequências de ensino investigativo.

\section{AGRADECIMENTOS}

Ao Programa Institucional de Bolsa de Iniciação à docência (PIBID), concedido pela Coordenação de Aperfeiçoamento de Pessoal de Nível Superior (CAPES) pertencente à FFCLRP da USP e à Fundação de Amparo à Pesquisa do Estado de São (FAPESP).

\section{REFERÊNCIAS BIBLIOGRÁFICAS}

1. Almeida LS. Facilitar a aprendizagem: ajudar os alunos a aprender e a pensar. Psicologia Escolar e Educacional. 2002;(6):155-65.

2. Boruchovitch E. Estratégias de aprendizagem e desempenho escolar: considerações para a prática educacional. Psicologia: Reflexão e Crítica. 1999;(12):361-376.

3. Cachapuz A, Perez DG, Carvalho AMP de. A Necessária Renovação do Ensino de Ciências. Editora Cortez, São Paulo; 2005. 264 p.

4. Carvalho DC, Quinteiro J. A formação docente e o PIBID: dilemas e perspectivas em debate. EntreVer, Florianópolis, v. 3, n. 4, p. i-xii, jan./jun. 2013. Acesso em 21 ago. 2014.

5. Del Prette A, Del Prette ZAP. Psicologia das relações interpessoais: Vivências para o trabalho em grupo. Petrópolis, RJ: Vozes; 2001. 231 p.

6. Del Prette AE, Del Prette ZAP. Habilidades Sociais e dificuldades de aprendizagem: Teoria e Pesquisa sob um enfoque multimodal. In: Del Prette A \& Del Prette ZAP (Org.), Habilidades sociais, desenvolvimento e aprendizagem: Questões conceituais, avaliação e intervenção; 2003. $314 \mathrm{p}$. 
7. Del Prette AE, Del Prette ZAP. Inventário de Habilidades Sociais para Adolescentes (IHSA-DelPrette): manual de aplicação, apuração e interpretação. São Paulo: Casa do Psicólogo; 2009. 54 p.

8. Kavale KA, Forness SR. Social skills deficits and learning disabilities: Ameta-analysis. Journal of Learning Disabilities. 1996;(29):226-237.

9. Osborne J, Duschl RE, Fairbrother R. Breaking the Mould? - Teaching Science for Public Understanding, The Nuffield Foundation, Londres; 2002. 92 p.

10. Oliveira KL, Boruchovitch E, Santos AAA. Escala de Estratégias de Aprendizagem: estudo das propriedades psicométricas. Em C. Machado, L. Almeida, M. A. Guisande, M. Gonçalves \& V. Ramalho (Orgs.), XI Conferência Internacional - Avaliação Psicológica: formas e contextos. Braga: Psiquilibrios Edições; 2006. p. 509-516.

11. Loranger AL.The study strategies of successful and unsuccessful high school students. Journal of Reading Behavior. 1994;(26):347-360.

12. Pozo JJ. Estrategias de Aprendizagem. Em C. Coll, J.Palacios \& A. Marchesi, (Orgs.), Desenvolvimento psicológico e educação: psicologia da educação. Porto Alegre: Artes Médicas; 1996. $197 \mathrm{p}$.

13. Reay D. I'm not seen as one of the clever children: Consulting primary school pupils about the social conditions of learning. Educational Review. 2006;(58):171-181.

14. Santos PL, Graminha SSV. Problemas emocionais e comportamentais associados ao baixo rendimento acadêmico. Estudos de Psicologia. 2006;(1):101-109.

15. Sasseron LH, Carvalho AMP. Alfabetização Científica: uma revisão bibliográfica. In: Revista Investigações em Ensino de Ciências. 2011;(16):59-77. 